\begin{tabular}{|c|c|}
\hline Title & A chaotic vibration energy harvester using magnetic material \\
\hline Author(s) & Sato, Takahiro; Igarashi, Hajime \\
\hline Citation & $\begin{array}{l}\text { Smart materials and structures, 24(2), } 25033 \\
\text { https://doi.org/10.1088/0964-1726/24/2025033 }\end{array}$ \\
\hline Issue Date & 2015-01-23 \\
\hline Doc URL & http:/hdl.handle.net/2115/60530 \\
\hline Rights & $\begin{array}{l}\text { This is an author-created, un-copy edited version of an article accepted for publication in Smart materials and structures. } \\
\text { The publisher is not responsible for any errors or omissions in this version of the manuscript or any version derived } \\
\text { from it. The V ersion of Record is available online at http://doi.org/10.1088/0964.1726/24/2025033 }\end{array}$ \\
\hline Type & article (author version) \\
\hline File Information & chaosFullIOP_verFin.pdf \\
\hline
\end{tabular}

Instructions for use 


\title{
A Chaotic Vibration Energy Harvester Using Magnetic Material
}

\author{
Takahiro Sato ${ }^{1,2}$, and Hajime Igarashi ${ }^{1}$ \\ ${ }^{1}$ Graduate School of Information Science and Technology, Hokkaido University, \\ Kita 14, Nishi 9, Kita-ku, Sapporo, 060-0814, Japan. \\ ${ }^{2}$ JSPS research Fellow. \\ E-mail: tsato@em-si.eng.hokudai.ac.jp
}

\begin{abstract}
This paper presents a new wideband electromagnetic vibration energy harvester (VEH) composed of a magnetic core embedded into the coil axis. The magnetic core generates a nonlinear magnetic force, which gives rise to the nonlinearity in the behavior of the VEH. Moreover, the magnetic core increases the flux linkage with the coil. These features improve the operational bandwidth and output power of the VEH. Numerical analysis and experimental measurements reveal that the operational bandwidth of the proposed VEH is over $30 \mathrm{~Hz}$ in which the output power is kept about $0.1 \mathrm{~mW}$. Moreover, the proposed VEH operates by complicated oscillation due to nonlinear forces acting on the oscillator. Evaluation of the Lyapunov exponent for the measured oscillation suggests that the proposed VEH produces chaotic oscillation.
\end{abstract}

\section{Introduction}

Energy harvesting has been receiving much attention as a voltage source for low-power electronic devices. So-called harvesting devices make it possible to create self-powered systems. In particular, the harvesting devices can be effectively used in wireless sensor networks for monitoring of structures such as bridges and tunnels [1-3]. Wireless sensor nodes with the harvesting devices can work for a long time without having to replace their batteries. Hence, the energy harvesting can realize wireless sensor systems with a large number of sensor nodes, which are suitable for monitoring the "health" of structures. The electromagnetic vibration energy harvester (VEH) [e.g. 4, 5] transforms vibration energy into electrical energy through magnetic induction. The VEHs basically consist of permanent magnets and pick-up coils. The magnets, which are oscillated by ambient vibrations, are placed on a cantilever. As a result, the magnetic flux across the coil temporally changes, and an electromotive force is induced.

Conventional VEHs produce the electrical energy through linear spring-damper oscillations. The maximum output power is thus generated when the ambient vibration matches the natural frequencies of a VEH. Linear VEHs are designed to have a high Q-factor in order to increase the output power at resonance. However, the ambient vibration in real constructions usually has very complicated vibration modes with a wide frequency spectrum [6]. It is clear that linear VEHs can absorb very little energy from such vibrations. To make effective energy harvesting from real-world vibrations, VEHs should have a wide frequency response. 
Various approaches to create a wideband VEH have been reported. For example, the frequency range of a VEH has been effectively expanded by adaptive tuning of the mechanical and electrical resonant frequencies of the oscillator [7, 8]. Multi-modal oscillators [9] and arrayed-harvesters [10], in which the VEHs have multiple resonant frequencies, have also been developed. The aim of these approaches is to increase the bandwidth of a linear VEH. Moreover, wideband operation is made possible by utilizing nonlinear oscillations [11-13]. Especially, the bistable VEH, which has a double-well potential, has recently been receiving much attentions [14-18]. In the bistable VEHs, the inertial mass of the device moves between two equilibrium positions when the oscillator can overcome the potential barrier by the vibrations regardless of frequency. For this reason, the bistable VEHs have the potential for broadband operation. Furthermore, it has been shown that bistable VEHs can harvest electrical power under noise excitations.

In this paper, a new wideband VEH which functions by nonlinear oscillation is presented. The proposed VEH contains a magnetic core embedded into the axis of a coil, which strengthens the magnetic force acting on the oscillator. This magnetic force gives rise to the nonlinearity in the behavior of the proposed VEH, through which the bandwidth is broadened. In addition, introducing the magnetic core increases in the flux linkage in the coil and, thus, increases output voltage. The behavior of the proposed VEH is numerically analyzed, and the performance of a manufactured VEH is experimentally evaluated. The experimental results show that the operational bandwidth of the proposed VEH is over $30 \mathrm{~Hz}$, in which the output power is kept to about $0.1 \mathrm{~mW}$. In addition, according to both analysis and measurement results, the oscillation of the proposed VEH is found to become complicated in a certain frequency range. The evaluation of Lyapunov exponent for the measured oscillation suggests that the proposed VEH has chaotic vibration in the above-mentioned frequency range.

\section{Vibration energy harvester with magnetic core in coil bobbin}

\subsection{Design concept}

The electromotive force (EMF) $V$ induced in VEHs is equal to the time derivative of the magnetic flux across the coil $\Phi$ : that is,

$$
V=\frac{\mathrm{D} \Phi}{\mathrm{D} t}=\frac{\mathrm{D}}{\mathrm{D} t} \iint_{S_{C}} \boldsymbol{B} \cdot \boldsymbol{i}_{S} d S,
$$

where $\boldsymbol{B}$ is magnetic induction, $S_{C}$ is the cross-sectional area of the coil, and $\boldsymbol{i}_{s}$ is the normal vector of $S_{C}$, respectively. In addition, $\mathrm{D} / \mathrm{D} t$ denotes the Lagrange derivative. In (1), for simplicity, the coil volume and number of turns are not considered. It is clear from (1) that $V$ increases with $\boldsymbol{B}$. One of the effective designs to increase $\boldsymbol{B}$ is to set opposing magnets into the coil [19]. On the other hand, magnetic cores are widely used in electric machinery such as transformers and reactors to increase magnetic flux. This strategy is also effective for VEHs. By introducing magnetic material into the coil axis, the flux across the coil is effectively increased. As the magnetic core for VEHs, soft magnetic composite (SMC) core [20], which consists of compressed iron-powders coated by electrically insulating material, is employed. Because SMC cores are suitable for the milli- and micro-scaled manufacturing and have relatively large saturated flux density (over 1T), they are suitable for VEHs. The flux distributions in a VEH model with and without a SMC core are shown in figures 1(a) and 1(b), respectively. The flux distributions are computed by the finite element method (FEM), which was used for all numerical computations performed in this study. According to figures 1(a) and 1(b), $\Phi$ is $0.97 \mathrm{mWb}$ for the VEH with the SMC core and $0.56 \mathrm{mWb}$ for the VEH without the SMC. These results suggests that introducing the SMC core effectively increases $\Phi$.

When the SMC core is introduced into the coil, an attraction magnetic force between the SMC core and the magnets is generated. If this magnetic force is nonlinear with respect to displacement, the 
VEH can function by nonlinear oscillations. In short, it is expected that the SMC core not only improves the efficiency but also broadens the bandwidth of the VEHs.

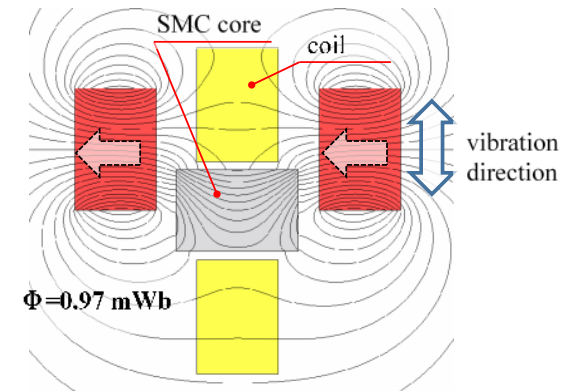

(a): with SMC core

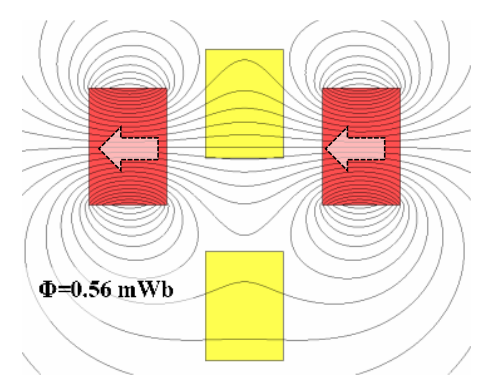

(b): without SMC core

Figure 1. Flux distributions in VEH with and without SMC core.

\subsection{Proposed VEH structure}

As for the design of the proposed $\mathrm{VEH}$, as shown in figure 2, the SMC core is introduced into the axis hole of the coil. Wire with diameter of $0.1 \mathrm{~mm}$ is wound around the SMC core. The number of turns in the coil is 600 . Phosphor-bronze is used for the cantilever material with size of $6 \times 9 \times 0.2 \mathrm{~mm}^{3}$. Effective spring constant of the cantilever, $k$, is $410 \mathrm{~N} / \mathrm{m}$. The cantilever is connected to four magnets and their keeper, that is, which are the oscillator of the proposed VEH. The total mass of the oscillator is $3.3 \mathrm{~g}$.

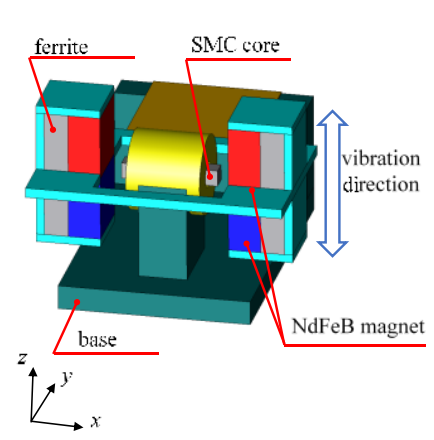

(a): 3D overview

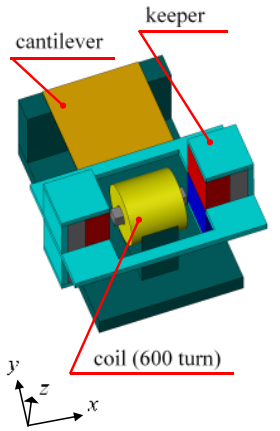

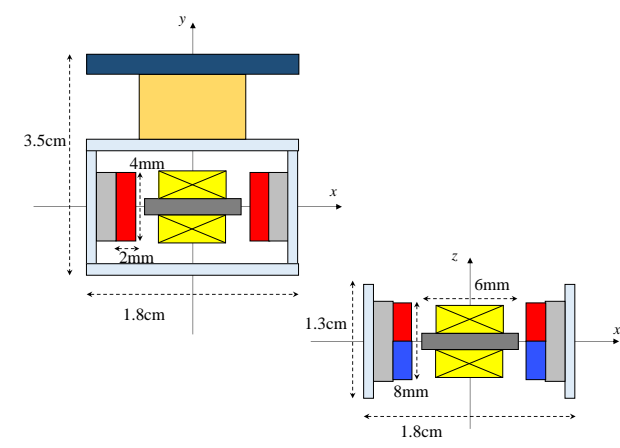

(b): size specifications

Figure 2. Proposed vibration energy harvester with SMC core in coil bobbin.

In the proposed VEH, the effective flux path is designed so as to increase the flux linkage with the coil. Furthermore, as shown in figure 3, the direction of the flux in the core dynamically changes in accordance with the relative displacement of the coil from the magnets. The computed EMF in the case with a the SMC core, when the magnets move from $2 \mathrm{~mm}$ to $-2 \mathrm{~mm}$ at $0.15 \mathrm{~m} / \mathrm{s}$, is shown in figure 4. For the comparison, the computed EMF values in the cases without any magnetic core and with a ferrite core, which is widely used for electric devices such as inductors and magnets, are also shown in figure 4. It is clear that the EMF with the SMC core is larger in comparison with those of the other cases. This result clearly suggests the superiority of the SMC cores.

The proposed VEH generates a magnetic force between the SMC core and magnets. The computed magnetic force acting on the oscillator against the relative displacement of the magnets and coil is shown in figure. 5. It is clear from the figure that the magnetic force and spring force act in opposite directions, and the magnetic force is nonlinear with respect to the displacement. As a result, as shown in figure 6, the total force acting on the oscillator is nonlinear. It is expected that this nonlinearly results in a wide operational frequency range for power generation of the proposed VEH. 


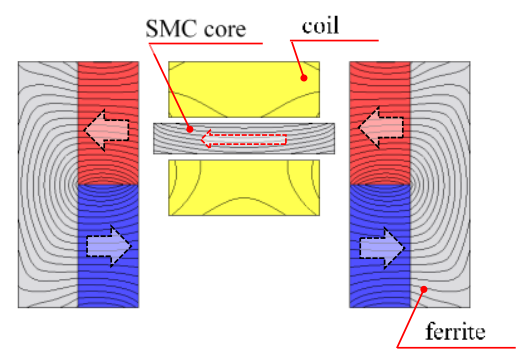

(a): positive displacement

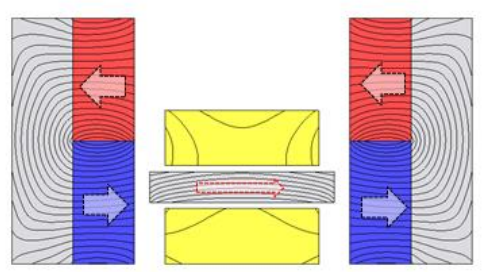

(b): negative displacement

Figure 3. Directions of flux in SMC core.

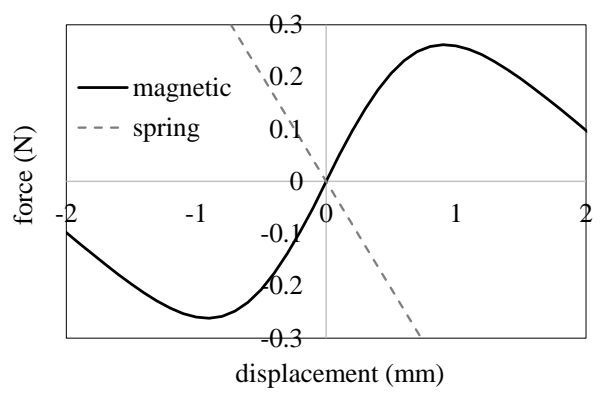

Figure 5. Magnetic force acing on oscillator.

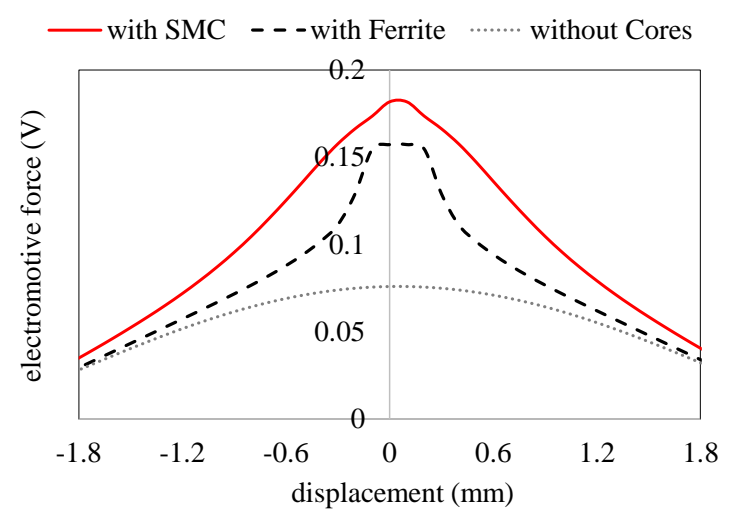

Figure 4. Electromotive force induced in coil when moving at $0.15 \mathrm{~m} / \mathrm{s}$.

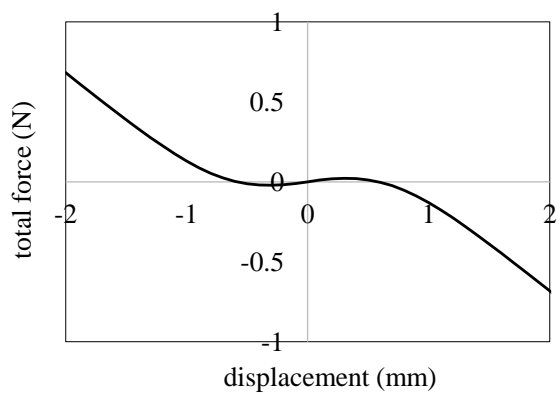

Figure 6. Total force (i.e. sum of magnetic and spring forces.)

\subsection{Relation to bistable VEH}

The relation between the proposed VEH and bistable VEH is investigated as follows. Bistable VEHs are characterized by a unique double-well potential, which is usually realized through repulsion or attraction forces between magnets [18]. Similarly, the proposed VEH also generates the attraction magnetic force between the magnets and the SMC core. This similarity suggests that the proposed VEH has a bistable structure.

To show the relation between the proposed VEH and bistable VEH, the potential energy of the proposed VEH is analyzed. The potential energy $E(X)$ is given by

$$
E(X)=E_{\text {mag }}(X)+\frac{1}{2} k X^{2},
$$

where $X$ represents the relative displacement between the magnets and coil, $E_{m a g}(X)$ is the magnetic energy stored in all the components of the VEH and surrounding air region, which is computed by FEM as follows:

$$
E_{\text {mag }}(X)=\iiint_{V} E_{m}(X) \mathrm{d} V,
$$




$$
E_{m}(X)= \begin{cases}\frac{\left\|\boldsymbol{B}-\boldsymbol{B}_{r}\right\|^{2}}{2 \mu} & \text { permanent magnets [21], } \\ \int_{0}^{\|\boldsymbol{B}\|}\|\boldsymbol{H}(b)\| \mathrm{d} b & \text { otherwise, }\end{cases}
$$

where $E_{m}(X)$ denotes magnetic energy density.

The computed potential energy is shown in figure 7, which shows that the proposed VEH has a double-well potential structure. Accordingly, the proposed VEH can be categorized as bistable VEH. It is therefore expected to operate across a wide bandwidth.

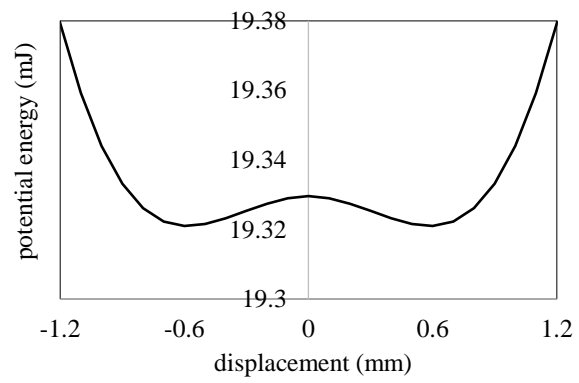

Figure 7. Potential energy of proposed VEH.

\section{Experiments}

\subsection{Numerical analysis}

The behaviour of the proposed VEH is analyzed by the numerical method based on the following spring-damper equation with magnetic force:

$$
m \ddot{X}+c \dot{X}+k X=F(X)+m \omega^{2} Y \sin (\omega t),
$$

where $m, c$, and $k$ are mass of the oscillator, a mechanical damping constant, and a spring constant, respectively, and $Y \sin (\omega t)$ is input vibration. In addition, $F(X)$ is the magnetic force acting on the oscillator which is approximately expressed by a polynomial of $X$. The coefficients in the polynomial are determined by the least-squares method to fit the magnetic force computed by FEM shown in figure 5. Note here that the spring-damper model (5) expresses the essential property of the system, although it is not be very accurate because the magnetic force also depends on the current path in the coil. It is assumed that the influence of the coil current on $F(X)$ is negligible.

Equation (5) is numerically solved using the Runge-Kutta method by applying a sinusoidal input, in which $m$ and $k$ are set to $3.3 \mathrm{~g}$ and $410 \mathrm{~N} / \mathrm{m}$, respectively, and $c$ is assumed to be 0.05 . Moreover, acceleration level $\omega^{2} Y$ is fixed to $1.0 \mathrm{~g}$ for all the frequencies. Amplitude of displacement, $X_{\mathrm{R} M S}$, against input frequency is plotted against input frequency in figure 8. A broad peak in $X_{\text {RMS }}$ appears in the frequency range from $20 \mathrm{~Hz}$ to about $50 \mathrm{~Hz}$. It can therefore be expected that the main output power is obtained in this frequency range. Time variations of $X$ and the trajectories on the phase plane

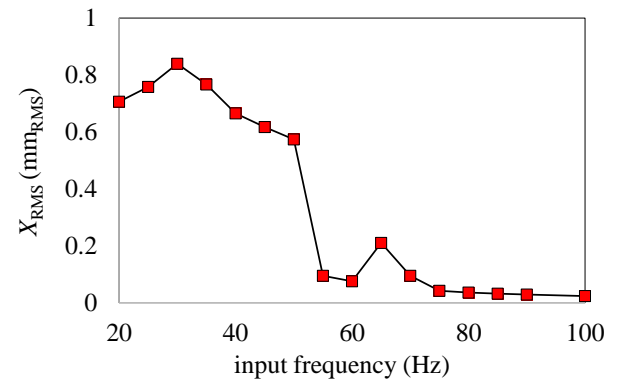

Figure 8. Computed displacement $X$ in RMS against frequency. 


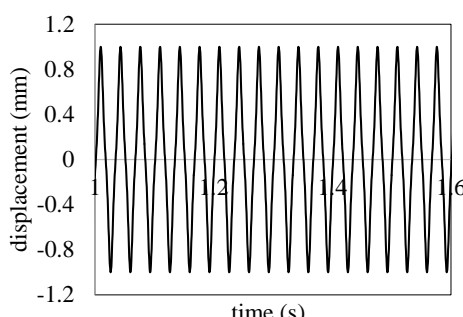

(a): $20 \mathrm{~Hz}$

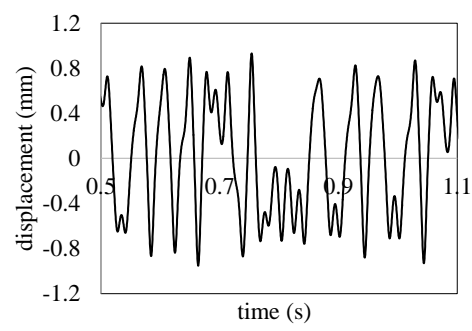

(b): $40 \mathrm{~Hz}$

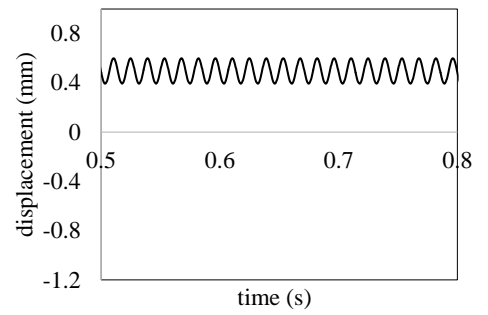

(c): $60 \mathrm{~Hz}$

Figure 9. Computed time variation of $X$.

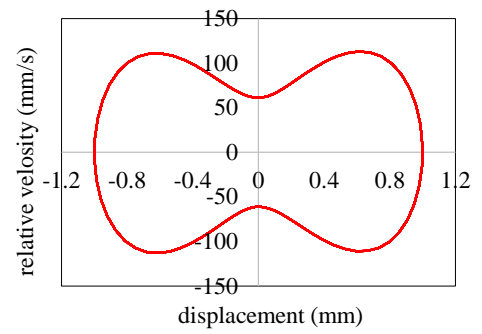

(a): $20 \mathrm{~Hz}$

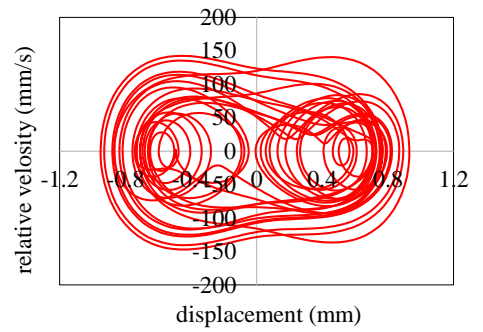

(b): $40 \mathrm{~Hz}$

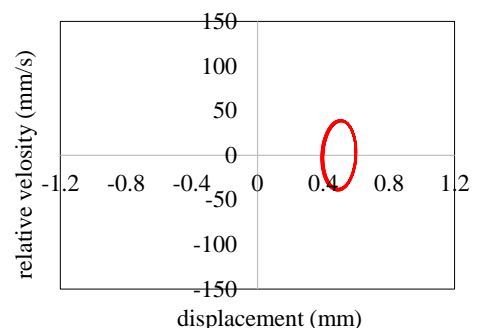

(c): $60 \mathrm{~Hz}$

Figure 10. Computed trajectories on phase plane of $X$ and its velocity.

of $X$ and its velocity are shown in figures 9 and 10, respectively. These figures show that the behaviour of the proposed VEH changes significantly with input frequency. In the range between $20 \mathrm{~Hz}$ and $40 \mathrm{~Hz}$, the proposed VEH operates by the interwell oscillations, in which the oscillator travels between two equilibriums. Moreover, the motion at $40 \mathrm{~Hz}$ is found to be complicated in comparison with that at other frequencies. On the other hand, the amplitude of $X$ at $60 \mathrm{~Hz}$ is small. It is thus concluded that the VEH operates by the intrawell oscillation. This is because the oscillator cannot overcome the potential barrier at this frequency. These analysis results correspond well with previously reported behaviours of bistable VEHs [18].

\subsection{Measurements}

\subsubsection{Measurement overview}

The proposed VEH was manufactured as shown in figure 11. The set up for measuring oscillation and output of the VEH is shown in figure 12. Sinusoidal vibration was applied to the base of the proposed $\mathrm{VEH}$, and the acceleration level was fixed to $0.6 \mathrm{~g}$ at all the frequencies. The time variation of the oscillator displacement was measured by a position sensor. A resistive load of $300 \Omega$ was connected to the coil, and the load voltage was measured by oscilloscope. Because the main purpose of this measurement is to reveal the output characteristic against frequency, impedance matching between the VEH and circuits was not considered.

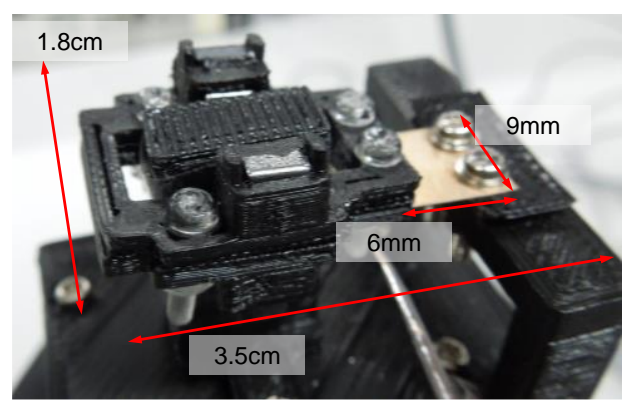

Figure 11. Manufactured proposed VEH.

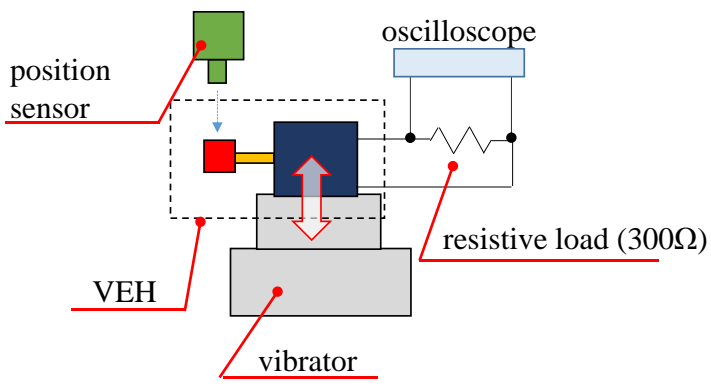

Figure 12. Measurement set-up. 


\subsubsection{Output power characteristics}

The displacement of the oscillator and the load voltage were measured for three times, and their average amplitudes, $X_{\mathrm{RMS}}$ and $V_{\mathrm{RMS}}$, were computed. The measured $X_{\mathrm{RMS}}$ and $V_{\mathrm{RMS}}$ are shown in figures 13 and 14, respectively. From figure 13, we can see that the measured response is laterally shifted in comparison with analyzed response shown in figure 8. In addition, double peaks appear in figure 13. One of reasons for the differences between analysis and measurement is thought to be due to manufacturing error. In the numerical analysis, the reference positions of magnets and coil are assumed to be equivalent. However, in the manufactured VEH, the reference position of the coil is not equal to that of the magnets. This manufacturing error causes the change in the potential energy shown in figure 7. As a result, the measured frequency response does not perfectly matches the numerical results. It is found from figure 14 that, within the range between $40 \mathrm{~Hz}$ to $70 \mathrm{~Hz}, V_{\mathrm{RMS}}$ is over $0.1 \mathrm{~V}_{\text {rms }}$, in which power consumption of the load is about $0.1 \mathrm{~mW}$. Namely, the bandwidth for power generation is over $30 \mathrm{~Hz}$. In that range, the output power is kept almost constant.

Time variations of $X$ and $V$ are shown in figure 15. Three distinct behaviors that depend on input frequency can be seen. The motion of the proposed VEH becomes complicated as frequency increases, as predicted by the numerical analysis mentioned in Section 3.1 . At $80 \mathrm{~Hz}$, the amplitude of the oscillation is much smaller than that at $60 \mathrm{~Hz}$. It is thus concluded that the behavior at $80 \mathrm{~Hz}$ corresponds to the intrawell oscillation.

These measurement results qualitatively agree with the numerical results. Hence, it is concluded that the proposed VEH has a bistable structure by which the bandwidth for power generation is broadened.

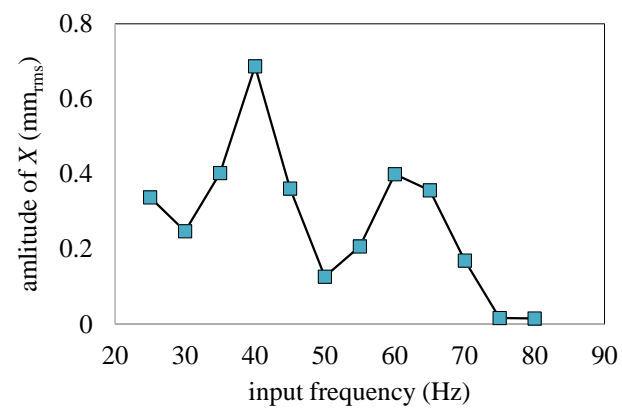

Figure 13. Measured displacement.

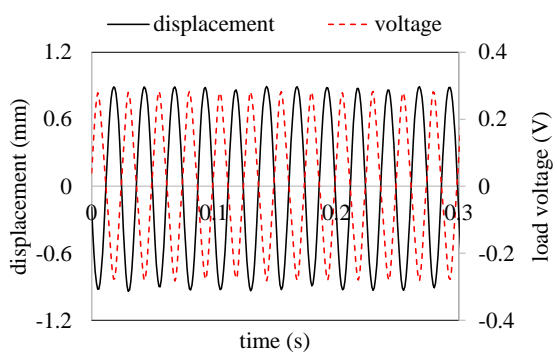

(a): $40 \mathrm{~Hz}$

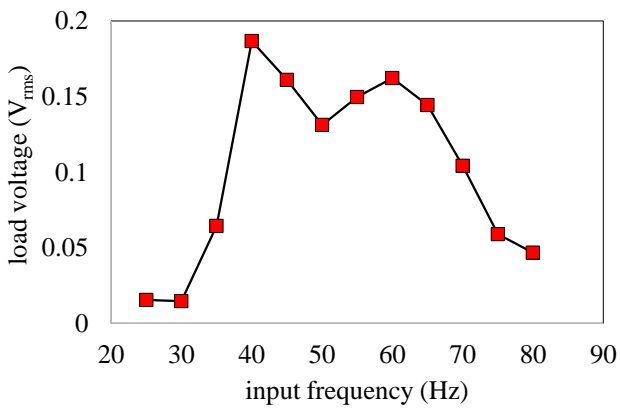

Figure 14. Measured load voltage.

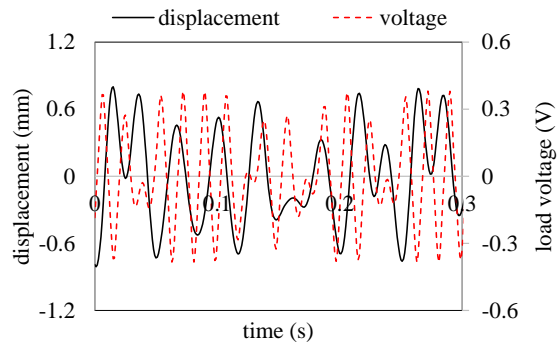

(b): $60 \mathrm{~Hz}$

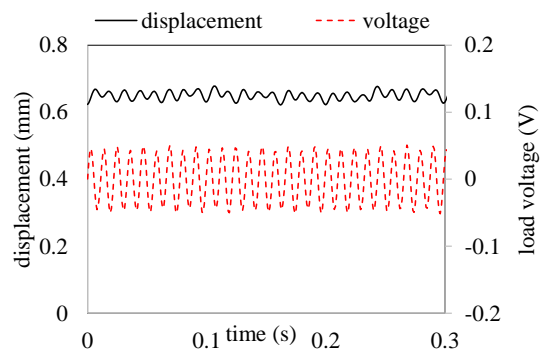

(c): $80 \mathrm{~Hz}$

Figure 15. Measured time variations of $X$ and $V$. 


\subsubsection{Complexity analysis}

To clarify the property of the observed complicated motion, the complexity of the oscillation is evaluated. The trajectories for $X$ are reconstructed based on the method of delay [22] in which the embedding dimension and delay $\tau$ are set to 6 and $0.4 \mathrm{~ms}$, respectively. The reconstructed attractors from $X$ are plotted in figure 16, according to which the reconstructed attractors at $40 \mathrm{~Hz}$ and $60 \mathrm{~Hz}$ show different characteristics. At $60 \mathrm{~Hz}$, the attractor is similar to the Lorentz attractor whose behavior is chaotic. To analyze the complexity of the trajectories, the Lyapunov exponent, $\lambda$, is evaluated using the Rosenstein method [23]. A positive $\lambda$ corresponds to the chaotic oscillation, whereas negative or zero $\lambda$ corresponds to the non-chaotic oscillations. However, when $\lambda$ is computed from measurement data, it is known that $\lambda$ results in positive even if the oscillations are non-chaotic because of the randomness due to noise. To reduce the noise effects, $\lambda$ is thus computed for 1000 times, and its average is taken. (Note here that the Rosenstein method uses a random number in its algorithm.) The averaged value of $\lambda$ is shown in figure 17 , which shows that the $\lambda$ values at $60 \mathrm{~Hz}$ and $65 \mathrm{~Hz}$ are clearly larger than those at the other frequencies. Histograms of $\lambda$ at $40 \mathrm{~Hz}$ and $60 \mathrm{~Hz}$ are shown in figure 18 , which shows that the distributions of $\lambda$ at $40 \mathrm{~Hz}$ and $60 \mathrm{~Hz}$ are statistically quite different. This result shows a statistical difference in the characteristics of their motions. Since $\lambda$ for the motion at $60 \mathrm{~Hz}$ is clearly positive, it strongly suggests that the motion at $60 \mathrm{~Hz}$ is chaotic.

The complexity of the oscillations can be clarified through the Lyapunov exponent. It can be seen from figure 17 that $\lambda$ increases with increasing frequency from $50 \mathrm{~Hz}$ to $65 \mathrm{~Hz}$, and then $\lambda$ drops at $70 \mathrm{~Hz}$. As shown in figures $15(\mathrm{c})$ and $16(\mathrm{c})$, the behavior of the $\mathrm{VEH}$ at $80 \mathrm{~Hz}$ corresponds to intrawell oscillations at which $\lambda$ takes a smaller value than that at $60 \mathrm{~Hz}$ and $65 \mathrm{~Hz}$. These results indicate that the motion characteristic of the VEH changes at $70 \mathrm{~Hz}$. In other words, the behavior of the proposed VEH becomes chaotic before the threshold frequency at which the motion transits to intrawell oscillation.

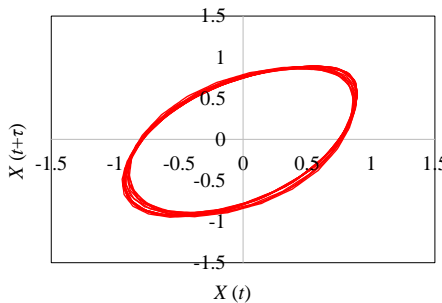

(a): $40 \mathrm{~Hz}$

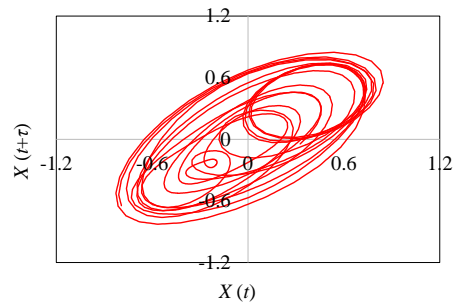

(b): $60 \mathrm{~Hz}$

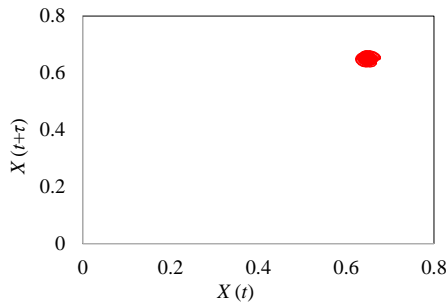

(c): $80 \mathrm{~Hz}$

Figure 16. Reconstructed attractors from $X$.

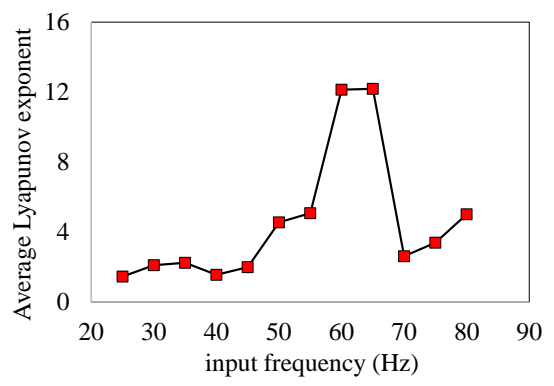

Figure 17. Average Lyapunov exponent for $X$.

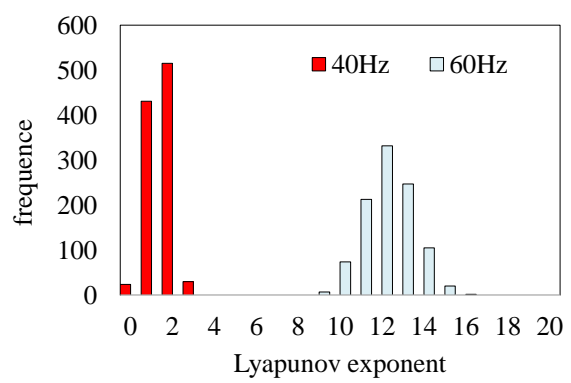

Figure 18. Histogram of $\lambda$.

\section{Conclusion}

The wideband VEH using an SMC core embedded in the axis hole of the coil has been proposed and fabricated. As for the proposed VEH, a nonlinear magnetic force between the magnets and SMC core 
creates a bistable structure. Moreover, the SMC core increases the flux linkage with the coil. The behavioural characteristic of the proposed VEH in regard to input frequency has numerically been analysed on the basis of the spring-damper equation with magnetic force. The analysis result shows a broad peak in displacement ranging from 20 to about $50 \mathrm{~Hz}$ occurs. It also shows that the proposed VEH has three distinct behaviours depending on the input frequency. The responses of the proposed VEH for $X$ and $V$ against frequency were measured. The measurement results show that operational bandwidth of the proposed $\mathrm{VEH}$ is over $30 \mathrm{~Hz}$ in which, within the range between 40 to $70 \mathrm{~Hz}, V_{\mathrm{RMS}}$ is over $0.1 \mathrm{~V}_{\text {rms }}$ at which power consumption of the load is about $0.1 \mathrm{~mW}$. The measured time variations of $X$ also shows three distinct behaviours depending on the input frequency. The trajectories for $X$ have been reconstructed, and the Lyapunov exponent for the reconstructed trajectories have been computed. From the Lyapunov exponent, it is suggested that the oscillations at $60 \mathrm{~Hz}$ and $65 \mathrm{~Hz}$ are chaotic. It is concluded that a wideband VEH with a bistable structure can be created by introducing a magnetic core into the coil to increase the induced electromotive force.

\section{Acknowledgement}

This study was supported by JSPS KAKENHI Grant Number 24310117, Grant-in-Aid for JSPS Fellows, and research grants from Japan Power Academy.

We would like to show our appreciation to Mr. Michiaki Suzuki and Dr. Kenji Kawano of TaiyoYuden Cor. who provided the SMC cores.

\section{References}

[1] E. Sazonov, H. Li, D. Curry, P. Pillay, 2009, Self-Powered Sensors for Monitoring of Highway Bridges, IEEE Sensors Journal, vo. 9, no. 11, pp. 1422-1429.

[2] S. Jang, et. al., 2010, Structural health monitoring of a cable-stayed bridge using smart sensor technology: deployment and evaluation, Smart. Mater. Struct., vol. 6, no. 5-6, pp. 439-459.

[3] M. Wischke, M. Masur, M. Kröner, P. Woias, 2011, Vibration harvesting in traffic tunnels to power wireless sensor nodes, Smart. Mater. Struct., vol. 20, no. 8, 085014.

[4] C. B. Williams, C. Shearwood, M. A. Harradine, P. H. Mellor, T. S. Birch, R. B. Yates, 2001, Development of an electromagnetic micro-generator, IEE Proc.-Circuits Devices Syst., Vol. 148, No. 6 pp. 337-342.

[5] S. P. Beeby, R. N. Torah, M. J. Tudor, P. G. Jones, T. O'Donnell, C. R. Saha, S. Roy, 2007, A micro electromagnetic generator for vibration energy harvesting, J. Micromech. Microeng., Vol. 17, no. 7 pp. $1257-1265$.

[6] S. Ju, et.al., 2013, A low frequency vibration energy harvester using magnetoelectric laminate composite, Smart Mater. Struct., vol 22, no. 11, 115037.

[7] C. Eichhorn, R. Tchagsim, N. Wilhelm, P. Woias, 2011, A smart and self-sufficient frequency tunable vibration energy harvester, J. Micromech. Microeng., vol. 21, no. 10, 104003.

[8] D. Zhu, S. Roberts, T. Mouille, M. J. Tudor, S. P. Beeby, 2012, General model with experimental validation of electrical resonant frequency tuning of electromagnetic vibration energy harvesters, Smart. Mater. Struct., vol. 21, no. 10, 105039.

[9] Y. Tadesse, S. Zhang, S. Priya, 2009, Multimodal Energy Harvesting System: Piezoelectric and Electromagnetic, J. Intell. Mater. Syst. Struct., vol. 20, no. 5, pp. 625-632.

[10] Z. Yang, J. Yang, 2009, Connected Vibrating Piezoelectric Bimorph Beams as a Wide-band Piezoelectric Power Harvester, J. Intell. Mater. Syst. Struct., vol. 20, no. 5, pp-569-574.

[11] L. G. W. Tvedt, D. S. Nguyen, E. Halvorsen, 2010, Nonlinear Behavior of an Electrostatic Energy Harvester Under Wide- and Narrowband Excitation, Journal of Microelectromechanical systems, Vol. 19, No. 2, pp. 305-316.

[12] B. P. Mann, N. D. Sims, 2009, Energy harvesting from the nonlinear oscillations of magnetic levitation, Journal of Sound and Vibration, vol 319, no. 1-2, pp. 515-530. 
[13] G. Sebald, H. Kuwano, D. Guyomar, B. Ducharme, 2011, Experimental Duffing oscillator for broadband piezoelectric energy harvesting, Smart. Mater. Struct., vol. 30, no. 20, 102001.

[14] S. C. Stanton, C. C. McGehee, B. P. Mann, 2010, Nonlinear dynamics for broadband energy harvesting: Investigation of a bistable piezoelectric inertial generator, Physica D: Nonlinear Phenomena, vol. 239, no. 10, pp. 640-653.

[15] B. P. Mann, B. A. Owens, 2010, Investigations of a nonlinear energy harvester with a bistable potential well, Journal of Sound and Vibration, vol. 329, no. 9, pp. 1215-1226.

[16] S. P. Beeby, L. Wang, D. Zhu, A. S. Weddell, G. V. Merrett, B. Stark, G. Szarka, B. M. AlHashimi, 2013, A comparison of power output from linear and nonlinear kinetic energy harvesters using real vibration data, Smart. Mater. Struct., vol. 22, no. 7, 075022.

[17] D. Zhu, S. P. Beeby, 2013, A broadband electromagnetic energy harvester with a coupled bistable structure, Journal of Physics: Conference Series, vol. 479, pp. 330-334.

[18] R. L. Harne, K. W. Wang, 2013, A review of the recent research on vibration energy harvesting via bistable systems, Smart. Mater. Struct., vol. 22, no. 2, 023001.

[19] V. R. Challa, S. Cheng, D. P. Amold, 2013, The role of coupling strength in the performance of electrodynamic vibrational energy harvesters, Smart. Mater. Struct., vol. 22, no. 2, 025005.

[20] H. Shokrollahi, K. Janghorban, 2007, Soft magnetic composite materials (SMCs), Journal of Materials Processing Technology, vol 189. no.1-3, pp. 1-12.

[21] H. C. Lovatt, P. A. Watterson, 1999, Energy Stored in Permanent Magnets, IEEE Trans. Magn., vol. 35 , no. 1, pp.505-507.

[22] F. Takens, 1981, Detecting strange attractors in turbulence, Lecture Notes in Mathematics, vol. 898, pp 366-381.

[23] M. T. Rosenstein, J. J. Collins, C. J. D. Luca, 1993, A practical method for calculating largest Lyapunov exponents from small data sets, Physica D: Nonlinear Phenomena, vol. 65, no. 1-2, pp. 117-134. 